\title{
Localisation of the melanocortin-2-receptor and its accessory proteins in the developing and adult adrenal gland
}

\author{
Rebecca J Gorrigan, Leonardo Guasti, Peter King, Adrian J Clark and Li F Chan \\ William Harvey Research Institute, Centre for Endocrinology, Queen Mary University of London, Barts and The London School of Medicine and Dentistry, \\ London, EC1M 6BQ, UK \\ (Correspondence should be addressed to L F Chan; Email: I.chan@ qmul.ac.uk)
}

\begin{abstract}
The melanocortin-2-receptor (MC2R)/MC2R accessory protein (MRAP) complex is critical to the production of glucocorticoids from the adrenal cortex. Inactivating mutations in either MC2R or MRAP result in the clinical condition familial glucocorticoid deficiency. The localisation of MC2R together with MRAP within the adrenal gland has not previously been reported. Furthermore, MRAP2, a paralogue of MRAP, has been shown in vitro to have a similar function to MRAP, facilitating MC2R trafficking and responsiveness to ACTH. Despite similar MC2R accessory functions, in vivo, patients with inactivating mutations of MRAP fail to be rescued by a functioning MRAP2 gene, suggesting differences in adrenal expression, localisation and/or function between the two MRAPs. In this study on the rat adrenal gland, we demonstrate that while MRAP and MC2R are highly expressed in the zona fasciculata, MRAP2 is expressed throughout the adrenal cortex in low quantities. In the developing adrenal gland, both MRAP and MRAP2 are equally well expressed. The MC2R/MRAP2 complex requires much higher concentrations of ACTH to activate compared with the MC2R/MRAP complex. Interestingly, expression of MC2R and MRAP in the undifferentiated zone would support the notion that ACTH may play an important role in adrenal cell differentiation and maintenance.
\end{abstract}

Journal of Molecular Endocrinology (2011) 46, 227-232

\section{Introduction}

Glucocorticoids are produced by the zona fasciculata $(\mathrm{ZF})$ of the adrenal cortex in response to ACTH stimulation of the melanocortin-2-receptor (MC2R), also known as the ACTH receptor. Ligand binding and activation of this $\mathrm{G}$ protein-coupled receptor principally results in stimulation of adenylyl cyclase, which catalyses the conversion of ATP to cAMP. Subsequent activation of protein kinase A leads to phosphorylation of cAMP response element protein, which in turn activates the transcription of genes involved in steroidogenesis (Lefkowitz et al. 1970, Lehoux et al. 1998).

It has emerged over recent years that the MC2R is dependent on the co-expression of an accessory protein, the MC2R accessory protein (MRAP; Metherell et al. 2005). The MC2R only responds to ACTH in heterologous cells when co-expressed with MRAP (Metherell et al. 2005, Roy et al. 2007, Sebag \& Hinkle 2007), and knockdown of MRAP in mouse adrenal Y1 cells (Cooray et al. 2008) and inactivating mutations in patients with familial glucocorticoid deficiency type 2 (FGD type 2; OMIM \#607398) (Metherell et al. 2005) lead to loss of ACTH responsiveness. It is also clear that MRAP mRNA is expressed in the adrenal gland
(Metherell et al. 2005). It is generally assumed that the glucocorticoid-producing fasciculata cell expresses both MC2R and MRAP, although this has not been formally established. Furthermore, MRAP has a single paralogue in the mammalian genome - MRAP2. MRAP and MRAP2 share a 39\% amino acid identity in the N-terminus and transmembrane domain (Chan et al. 2009). The MRAP2 gene is expressed in the brain and the adrenal gland. In vitro MRAP2 appears to have a qualitatively similar ability to MRAP, enabling MC2R trafficking from the endoplasmic reticulum to the cell surface and the formation of a functional ACTH receptor (Chan et al. 2009). However, studies with mouse or zebrafish MRAP2 did not show this effect when a lower concentration of ACTH was used (Sebag \& Hinkle 2009, Agulleiro et al. 2010). Since the presence of MRAP2 in the adrenal gland does not protect patients with MRAP mutations from developing glucocorticoid deficiency, the question arises as to whether MRAP and MRAP2 are expressed at different levels, in different cell types or whether they differ functionally to lower doses of ACTH. In this study, we analysed the quantitative expression, anatomical localisation and functional properties of these two MRAPs. 


\section{Materials and methods}

\section{Plasmid and probes}

Vectors used in the cAMP reporter assays, MRAP-flag and MRAP2-flag were cloned, as previously described (Chan et al. 2009). HA-MC2R was purchased from the Missouri S\&T cDNA Resource Center (University of Missouri at Rolla, Rolla, MO, USA). Templates for creating riboprobes for in situ hybridisation (ISH) were produced by inserting full-length Mrap (forward primer 5'-CTGTCCCGTTCACCAGCTAT-3', reverse primer $5^{\prime}$-TAACCATTGGTCAATGCCAG-3'), Mrap2 (forward primer $5^{\prime}$-CTGTGAAGGGCTCTCTGACC-3 ${ }^{\prime}$, reverse primer $5^{\prime}$-GAACACATCCTCAGGAAGGC-3'), or $M c 2 r$ (forward primer $5^{\prime}$-ATCTGCAGTTTGGCCATTTC-3' ${ }^{\prime}$, reverse primer 5'-ATGCCATTGACCTGGAAGAG-3') cDNA produced from rat adrenal RNA into pGEMT-Easy vector (Promega). All constructs were confirmed by sequencing.

\section{Nonradioactive-ISH and immunohistochemistry}

Adrenal glands from adult Wistar male rats were snap frozen, or fixed in 4\% paraformaldehyde and embedded in paraffin. Wistar rat embryos (embryonic day 17.5) were collected in ice-cold PBS (Sigma), fixed in $4 \%$ paraformaldehyde, cryoprotected in $30 \%$ sucrose and embedded in OTC compound (Fisher Scientific, Loughborough, UK). Digoxigenin (DIG)-labelled sense and antisense cRNA riboprobe production and nonradioactive-ISH (NR-ISH), or combined NR-ISH/immunohistochemistry (IHC), were performed as reported by Guasti et al. (2010). Briefly, after NR-ISH staining, sections were blocked with $10 \%$ normal goat serum and incubated overnight at room temperature with anti-CYP11B1 mouse monoclonal antibody (a gift of Dr C Gomez-Sanchez, University of Mississippi Medical Center, Jackson, MS, USA) diluted 1:20 in PBSTriton X-100 $0 \cdot 1 \%$. The sections were then washed and incubated for $2 \mathrm{~h}$ with goat anti-rabbit Alexa Fluor 488 secondary (Invitrogen) diluted 1:1000 in PBS and, after further washes, reacted with $4^{\prime}$,6-diamidino-2-phenylindole (DAPI; Sigma) for $1 \mathrm{~min}$. Images were captured using a Leica DC200 camera (Leica, Solms, Germany) equipped with a DCViewer software (Leica), imported into Photoshop (Adobe Systems), and bright field ISH images were converted into black and white, inverted and pseudo-coloured to red before merging them with the immunofluorescence images in RGB mode.

\section{Real-time quantitative RT-PCR}

cDNA was synthesised from rat total adrenal RNA (extracted from the adrenal glands of two adult male Wistar rats) by reverse transcription using M-MLV Reverse Transcriptase (Promega), following the manufacturer's guidelines. Gene expression levels were analysed by SYBR
Green assays, using KAPA SYBR Fast Universal qPCR kit (Anachem, Luton, UK) and a Stratagene thermocycler. Thermal profile used: $1 \times 95^{\circ} \mathrm{C}$ for $3 \mathrm{~min}, 40 \times\left(95^{\circ} \mathrm{C}\right.$ for $3 \mathrm{~s}, 55^{\circ} \mathrm{C}$ for $20 \mathrm{~s}$ and $72^{\circ} \mathrm{C}$ for $\left.1 \mathrm{~s}\right), 1 \times\left(95^{\circ} \mathrm{C}\right.$ for $1 \mathrm{~min}$, $55^{\circ} \mathrm{C}$ for $30 \mathrm{~s}$ and $95^{\circ} \mathrm{C}$ for $\left.30 \mathrm{~s}\right)$. Primers used for rat cDNA were Mrap (forward, 5'-ATTCTGCTCTACATGTCCTG-3' ${ }^{\prime}$, reverse, 5'-TACTTCCTGGCTCATCTG-3') and Mrap2 (forward, 5'-CAGAGGCTGACTTCTAACAG$3^{\prime}$, reverse, 5'-CAGAATCCAATCACGATG-3' ${ }^{\prime}$. Gene expression of the housekeeping gene Gapdh was used for normalisation (forward, $5^{\prime}$-TGCACCACCAACTGCTTAG-3' ${ }^{\prime}$, reverse, $5^{\prime}$-GGATGCAGGGATGATGTTC-3' ${ }^{\prime}$ ) for rat cDNA. Experiments were performed in duplicate and repeated three times. Data were analysed using MxPro software (Stratagene, Stockport, UK). The $C_{\mathrm{t}}$ value of the unknown was quantified absolutely by interpolating the quantity from a standard curve, whose quantities were pre-determined spectrophotometrically at OD $260 \mathrm{~nm}$ (NanoDrop ND-1000 spectrophotometer; Wilmington, DE, USA).

\section{Cell culture, transfection and cAMP reporter assay}

Human embryonic kidney (HEK293) cells were grown in DMEM (Sigma) containing $10 \%$ foetal bovine serum (Sigma) and penicillin/streptomycin (Invitrogen). Cells were grown to around $70-80 \%$ confluency in six-well plates and transfected with HA-MC2R, plus either MRAPflag, MRAP2-flag, MRAP-flag and MRAP2-flag or pcDNA $3.1(+)$ empty vector, along with $\alpha \mathrm{GSU}-846$ luciferase and pRL-CMV Renilla luciferase plasmid constructs, using Lipofectamine 2000 (Invitrogen), following the manufacturer's instructions. Twenty-four to forty-eight hours after transfection, cells were stimulated for $6 \mathrm{~h}$ with $\mathrm{ACTH}_{1-39}$ at concentrations of $10^{-4}$ to $10^{-11} \mathrm{M}$. Whole cell lysates were collected and assayed using the Dual-Luciferase Reporter Assay System (Promega). Luciferase activity was measured using a multiplate reader (LUMIstar Omega; BMG Labtech, Aylesbury, UK) and normalised to the pRL-CMV Renilla luciferase activity.

\section{Statistical analysis}

The data represent mean \pm s.E.M. of at least three independent experiments performed in duplicate. Prism software (La Jolla, CA, USA) was used to evaluate $\mathrm{EC}_{50}$ values. Statistical analysis was performed using Student's $t$-test.

\section{Results}

\section{Mrap, Mrap2 and Mc2r localisation in the adult and foetal rat adrenal gland}

ISH was performed to visualise the localisation of Mrap, Mrap2 and Mc2r mRNA expression within the 
adrenal gland (Fig. 1). Mrap and Mrap2 were readily detectable specifically in the adrenal gland during development (Fig. 1A-F). Mrap and Mrap2 NR-ISH was performed in combination with CYP11B1 IHC, which demonstrated that cortical, but not medullary cells, expressed $M c 2 r$ accessory proteins (Fig. $1 \mathrm{G}-\mathrm{G}^{\prime \prime}$ ).

In the adult adrenal gland, Mrap and $M c 2 r$ were expressed throughout the cortex (Fig. 1H-K), although their expression was weaker in the zona glomerulosa (ZG) compared with the ZF. In the ZF, a gradient was observed with higher levels of expression seen at the periphery decreasing towards the cortico-medullary boundary. When combined $M c 2 r$ NR-ISH/CYP11B1 IHC was performed, the inner layer of cells within the undifferentiated zone ( $\mathrm{ZU})$, also known as the zona intermedia, which was negative for CYP11B1, was strongly positive for $M c 2 r$ expression (Fig. 1L-L" $\mathrm{L}^{\prime \prime}$. Combined Mrap NR-ISH/CYP11B1 IHC revealed the same pattern of expression (data not shown). In contrast, Mrap2 was detected at low levels throughout all zones of the adrenal cortex (Fig. 1M).
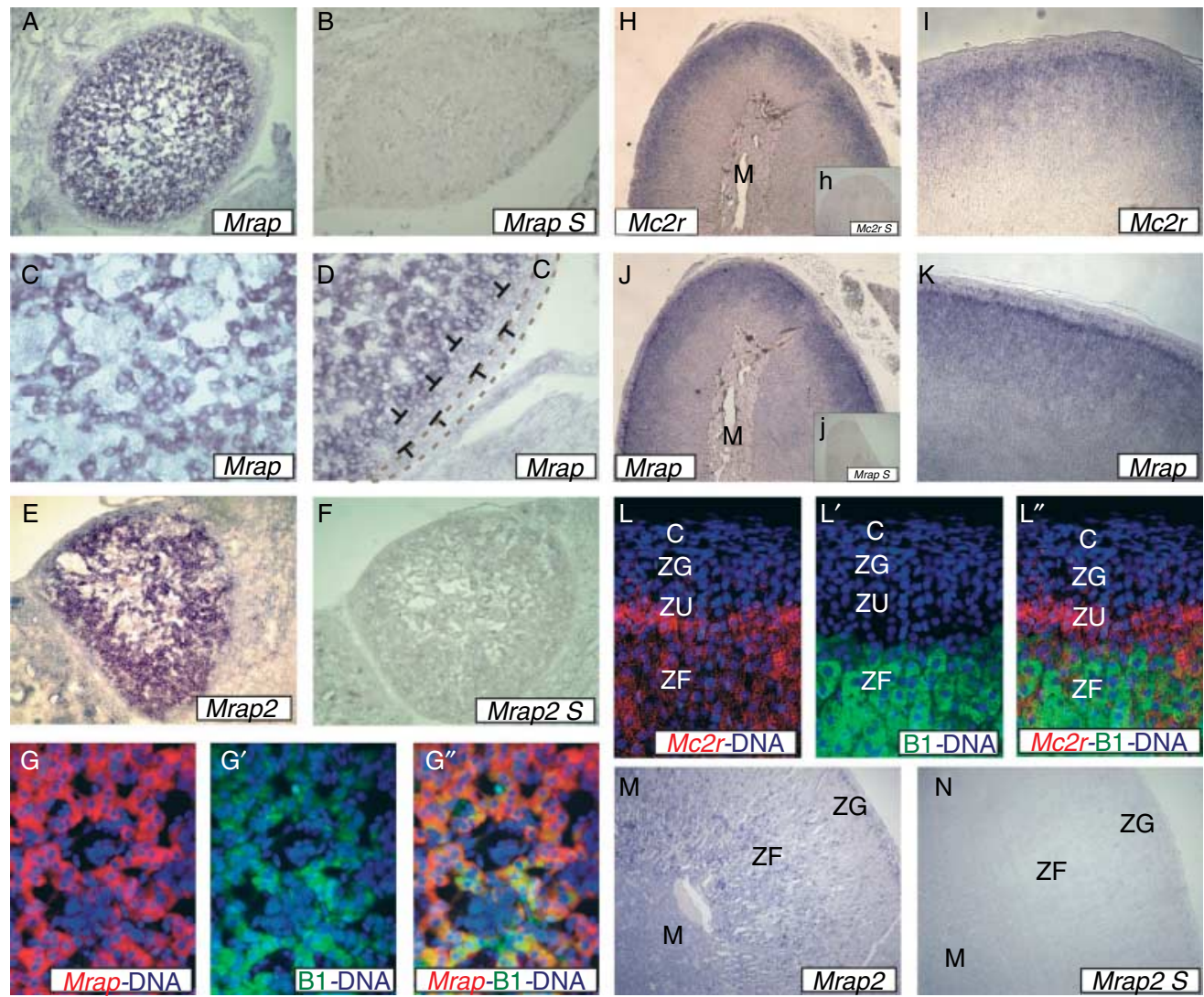

Figure 1 In situ hybridisation showing localisation of Mrap, Mrap2 and Mc2r in foetal and adult Wistar rat adrenal glands. (A-G) Foetal Wistar rat adrenal glands (embryonic age 17.5 days); $(\mathrm{H}-\mathrm{N}$ ) adult male Wistar rat adrenal glands. (A and B) Low-magnification images of foetal rat adrenal glands incubated with Mrap antisense and sense $(S)$ riboprobes. Mrap is present throughout the foetal rat adrenal gland. The sense probe resulted in no specific staining. (C and D) $10 \times$ magnified images of foetal rat adrenal glands. No Mrap staining in the adrenal capsule $(C)$ or subcapsular zone (indicated by $T)$. ( $E$ and F) Foetal rat adrenal glands incubated with Mrap2 antisense and sense (S) riboprobes. Mrap2 is present throughout the foetal rat adrenal gland. The sense probe resulted in no specific staining. (G) Combined Mrap ISH and CYP11B1 immunohistochemistry. Nuclei are stained with DAPI. Cells positive for Mrap also express CYP11B1, suggesting these cells are capable of glucocorticoid synthesis and are destined to localise to the zona fasciculata postnatally. $(\mathrm{H}-\mathrm{K}) \mathrm{Mc} 2 \mathrm{r}$ and Mrap are present in the zona fasciculata $(\mathrm{ZF})$, with relative sparing of the capsule, zona glomerulosa (ZG) and medulla (M). Expression was strongest at the periphery, with a gradient reduction towards the medulla. The sense probes resulted in no specific staining $(\mathrm{h}, \mathrm{j})$. $(\mathrm{L}) \mathrm{Combined}$ Mc2r ISH and CYP11B1 immunohistochemistry. Mc2r is expressed in the ZF, in cells co-expressing CYP11B1. Mc2r is also expressed highly in the undifferentiated zone, in cells which lack CYP11B1 expression. ( $\mathrm{M}$ and $\mathrm{N}$ ) Mrap2 is expressed at low levels throughout the adult rat adrenal gland. The sense probe resulted in no specific staining $(\mathrm{N})$. 


\section{Quantification of Mrap and Mrap2 in rat adrenal gland}

Quantitative PCR was performed on rat total adrenal gland cDNA for Mrap and Mrap2 expression. Results were expressed relative to Gapdh or copy numbers per reaction. Mrap was expressed at significantly higher levels compared with Mrap2 (18× greater than Mrap2 when expressed relative to Gapdh, $P<0 \cdot 0001$, and $21 \times$ greater when expressed as copy number per reaction, $P=0 \cdot 0015$; Fig. 2).

\section{Functional MC2R differences between MRAP and MRAP2}

HEK293 cells, which lack endogenous melanocortin receptors, were transfected with MC2R and either MRAP, MRAP2 or both. Stimulation with ACTH generated a dose-dependent increase in cAMP production, as assessed by the luciferase activity produced from the cAMP-responsive aGSU-846 luciferase reporter construct. Cells transfected with MC2R and MRAP2 were able to produce cAMP; however they required significantly higher concentrations of ACTH to achieve this (EC50 $1 \times 10^{-9}$ for MRAP and $9 \cdot 3 \times 10^{-7}$ for MRAP2; $\left.P<0 \cdot 0001\right)$. Cells expressing both MRAP and MRAP2 did not exhibit a reduction or augmentation of the MRAP/MC2R cAMP response to ACTH stimulation (Fig. 3).

\section{Discussion}

The importance of MRAP in MC2R function is exemplified by the clinical condition of FGD, in which disabling mutations of either gene results in life-threatening isolated glucocorticoid deficiency (Metherell et al. 2005). Although the expression of $M R A P$ has been demonstrated in the adrenal gland by RT-PCR, little is known about the localisation of MRAP mRNA within the different zones of the adrenal gland (Metherell et al. 2005). In addition, the presence of MRAP2 in the adrenal gland could indicate the potential for redundancy (Chan et al. 2009), although this is unlikely as patients with MRAP mutations are not rescued by an intact $M R A P 2$ gene.

Our ISH studies have shown that Mrap and $M c 2 r$ are highly expressed in the rat adrenal $\mathrm{ZF}$ in cells expressing CYP11B1 and thus being capable of glucocorticoid synthesis. The level of expression appears to be highest at the periphery, with a gradient reduction towards the medulla. Interestingly, the highest level of Mrap and $M c 2 r$ expression is in the inner part of ZU, directly adjacent to the ZF. The ZU lies between the $\mathrm{ZF}$ and $\mathrm{ZG}$ in the adult rat adrenal and has been postulated to contain stem cells that contribute to
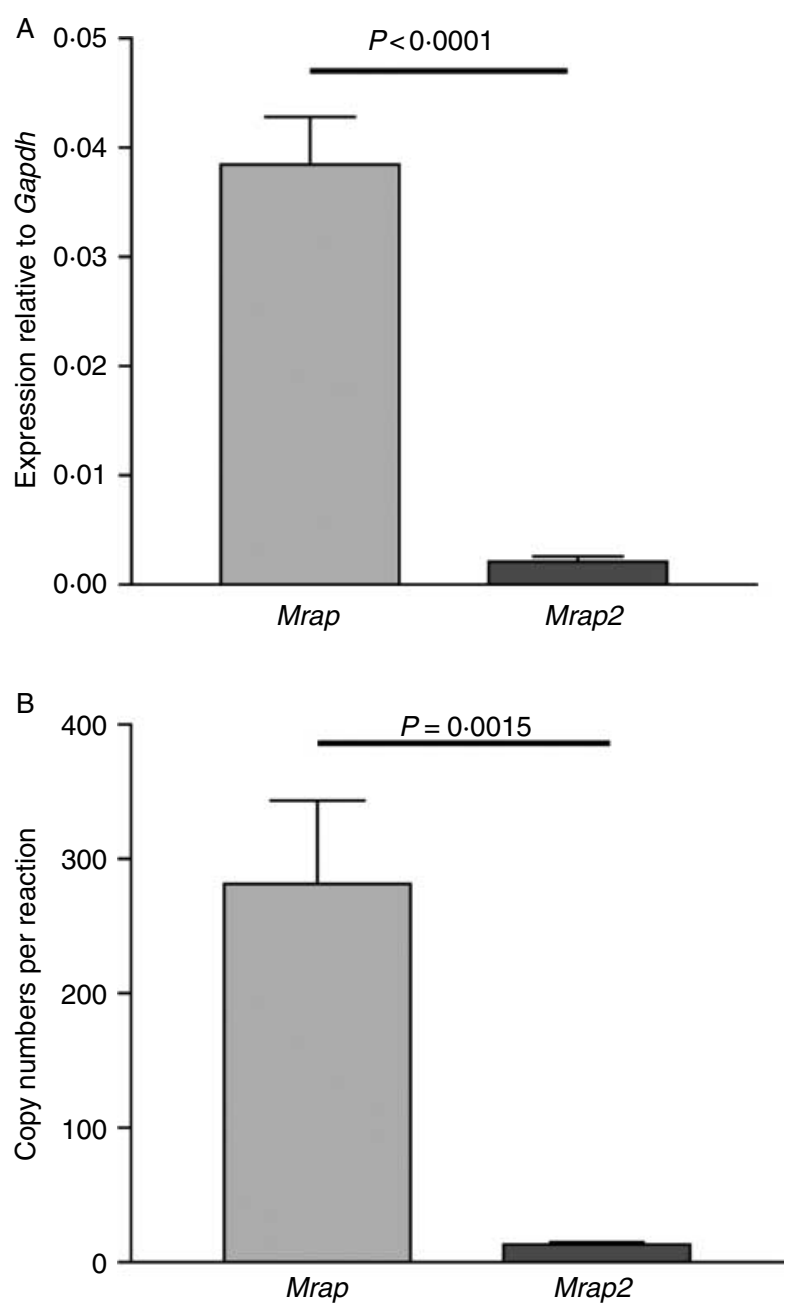

C

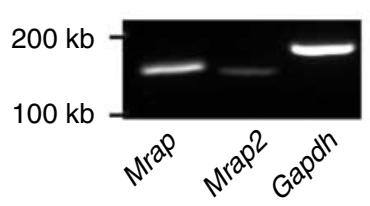

Figure 2 Quantification of Mrap and Mrap2 in rat adrenal gland. Expression of Mrap and Mrap2 in total adult Wistar rat adrenal glands $(A)$ normalised to Gapdh showing a 18-fold reduction in Mrap2 relative to $\operatorname{Mrap}(P<0.0001), n=3$. (B) Expressed as copy numbers per reaction showing a 21 -fold reduction in Mrap2 relative to Mrap $(P=0.0015), n=3$. (C) Agarose gel image demonstrating the expression of Mrap and Mrap2 in rat adrenal gland by RT-PCR.

steroidogenic cell maintenance (Wright \& Voncina 1977, Mitani et al. 2003). Expression of Mrap and Mc2r in the ZU in relatively undifferentiated, CYP11B1negative cells is interesting and suggests that ACTH may have a role in adrenal cell differentiation and maintenance. The concept that ACTH plays an 


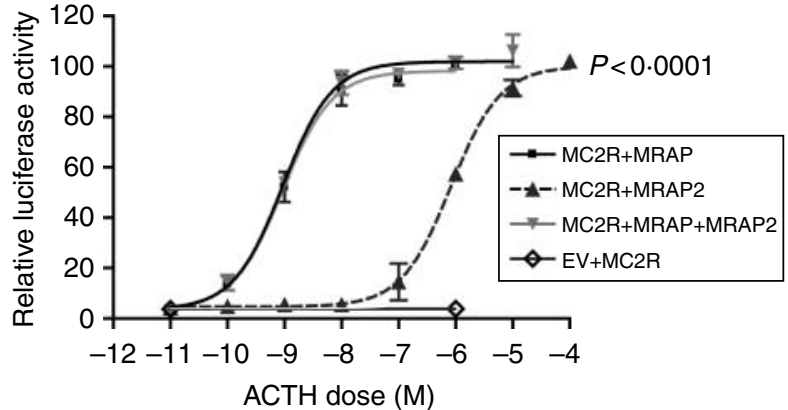

Figure $3 \mathrm{MC} 2 \mathrm{R}$ response to $\mathrm{ACTH}$ in the presence of MRAP or MRAP2 or both. HEK293 cells were transiently transfected with HA-MC2R, $\alpha$ GSU846-luc, pRL-CMV Renilla luciferase and either MRAP-Flag, MRAP2-Flag, MRAP-Flag and MRAP2-Flag or empty vector together with and stimulated with ACTH for $6 \mathrm{~h}$. Dose-response curves demonstrating a right shift in the case of MRAP2. EC50 $1 \times 10^{-9}$ for MRAP and $9.3 \times 10^{-7}$ for MRAP2; $P<0.0001$. EV, empty vector.

important role on adrenal growth and adrenal zonal maintenance has been proposed for some time (reviewed in Karpac et al. (2007) and Chan et al. (2011)). Hypophysectomised animal models have adrenocortical atrophy (Robinson et al. 1983) and similarly atrophied adrenal glands were noted in POMC-knockout mice (Coll et al. 2004, Karpac et al. 2005). The administration of ACTH alone appeared to restore adrenal weight, morphology and function to normal in these models (Robinson et al. 1983, Coll et al. 2004). The adrenal histology of FGD would support the suggestion that MC2R and/or MRAP may be important for adrenal zonal development as the glands from deceased patients demonstrate disorganisation of glomerulosa cells with almost complete absence of fasciculata and reticularis cells (Clark \& Weber 1998).

In comparison to the high expression levels seen with Mrap and Mc2r, ISH studies for Mrap2 showed very low levels of expression in adult rat adrenal but high expression in the developing adrenal gland. Real-time RT-PCR quantification of Mrap and Mrap2 expression in the adult rat adrenal gland demonstrated that Mrap expression is significantly greater than Mrap2 expression, supporting the low expression levels detected by ISH.

We previously showed that MRAP2 was able to rescue the expression and function of the MC2R in nonadrenal cells (Chan et al. 2009). Later studies, however, suggested that although MRAP2 was capable of assisting MC2R to the cell surface, the receptor was unresponsive to ACTH (Sebag \& Hinkle 2009, Agulleiro et al. 2010). Here, we demonstrate that MRAP2 does indeed enable functioning of the MC2R in response to ACTH stimulation in vitro; however, much higher concentrations of ACTH are required to generate the same amount of cAMP in the presence of MRAP2 compared with MRAP. The 1000-fold difference in $\mathrm{EC}_{50}$ provides an explanation and hence agreement for the different results obtained by other groups who utilised different ACTH doses for stimulation (Chan et al. 2009, Sebag \& Hinkle 2009, Agulleiro et al. 2010). It is likely that even in FGD type 2, where ACTH concentrations are often $>1000 \mathrm{pg} / \mathrm{ml}$ (Clark \& Weber 1998), this is still inadequate to stimulate MC2R function. This suggests that although MRAP2 can enable MC2R function, this is not its role in vivo.

Studies have shown that MRAP2 can heterodimerise with MRAP in vitro (Chan et al. 2009) and such heterodimers adopt an antiparallel conformation (Chan et al. 2009, Sebag \& Hinkle 2010). Western blotting of tissues has revealed band sizes of which are suggestive of homodimers rather than heterodimers (Cooray et al. 2008, Chan et al. 2009). Nevertheless, experiments on $\mathrm{Y} 1$ and $\mathrm{CHO}$ cells suggest that MRAP2 has an inhibitory role and competes with MRAP to bind the MC2R, reducing cAMP production in response to ACTH stimulation (Sebag \& Hinkle 2010) in vitro. In this study, we did not show any reduction in cAMP generation of the MRAP/MC2R complex with the addition of MRAP2. Likewise, another study on zebrafish MRAP2 did not report an inhibitory effect, but found that MRAP2 had additive effects on the MRAP/MC2R cAMP response to ACTH (Agulleiro et al. 2010). This may, however, represent the different cell lines used in the studies and hence different levels of expression achieved (Chan et al. 2009, Agulleiro et al. 2010, Sebag \& Hinkle 2010). While the hypothesis of a dominant-negative action of MRAP2 is an attractive one, it is difficult to envisage such a mechanism in view of the low levels of MRAP2 expression in adrenal tissue, the diffuse zonal localisation in the adrenal gland and the amount of MRAP2 expression that may be required to significantly shift the $\mathrm{EC}_{50}$ for this to be relevant. Whether MRAP2 expression in the foetal adrenal gland determines the tone of the hypothalamic-pituitary-adrenal axis in adult life has yet to be studied and can only be answered with the generation of knockout mice.

In conclusion, we have demonstrated that $M c 2 r$, Mrap and CYP11B1 are co-expressed in fasciculata cells in the rat adrenal gland, consistent with their role in ACTH stimulation of steroidogenesis. Furthermore, Mrap and Mc2r are expressed in some cells of the rat adrenal ZU, suggesting that ACTH may be important in adrenal cell differentiation and maintenance. The role of MRAP2 in the adrenal gland is unclear. Although MRAP2 is capable of facilitating MC2R function in response to ACTH stimulation, the concentration of ACTH required is much higher than that likely to be generated in vivo. However, the apparent high expression of MRAP2 in the developing adrenal gland may suggest a role in early development. 
Availability of global or tissue-specific Mrap2-knockout mice would certainly help clarify the role of this protein during development and in adult homeostasis.

\section{Declaration of interest}

The authors declare that there is no conflict of interest that could be perceived as prejudicing the impartiality of the research reported.

\section{Funding}

R J G is supported by a Wellcome Clinical Research Training Fellowship (grant number WT092024MA). L F C holds an MRC/ Academy of Medical Sciences Clinician Scientist Fellowship (grant number G0802796). L G is supported by an MRC project grant awarded to PJK (grant number G0700767).

\section{Acknowledgements}

The authors thank Dr C Gomez-Sanchez (University of Mississippi Medical Center, Jackson, Mississippi) for providing the CYP11B1 antibodies.

\section{References}

Agulleiro MJ, Roy S, Sanchez E, Puchol S, Gallo-Payet N \& Cerda-Reverter JM 2010 Role of melanocortin receptor accessory proteins in the function of zebrafish melanocortin receptor type 2. Molecular and Cellular Endocrinology 320 145-152. (doi:10.1016/ j.mce.2010.01.032)

Chan LF, Webb TR, Chung TT, Meimaridou E, Cooray SN, Guasti L, Chapple JP, Egertova M, Elphick MR, Cheetham ME et al. 2009 MRAP and MRAP2 are bidirectional regulators of the melanocortin receptor family. PNAS 106 6146-6151. (doi:10.1073/pnas. 0809918106)

Chan LF, Metherell LA \& Clark AJ 2011 Effects of melanocortins on adrenal gland physiology. European Journal of Pharmacology (In Press). (doi:10.1016/j.ejphar.2010.11.041)

Clark AJ \& Weber A 1998 Adrenocorticotropin insensitivity syndromes. Endocrine Reviews 19 828-843. (doi:10.1210/er.19.6.828)

Coll AP, Challis BG, Yeo GS, Snell K, Piper SJ, Halsall D, Thresher RR \& O'Rahilly S 2004 The effects of proopiomelanocortin deficiency on murine adrenal development and responsiveness to adrenocorticotropin. Endocrinology 145 4721-4727. (doi:10.1210/en.20040491)

Cooray SN, Almiro Do Vale I, Leung KY, Webb TR, Chapple JP, Egertova M, Cheetham ME, Elphick MR \& Clark AJ 2008 The melanocortin 2 receptor accessory protein exists as a homodimer and is essential for the function of the melanocortin 2 receptor in the mouse Y1 cell line. Endocrinology 149 1935-1941. (doi:10.1210/ en.2007-1463)
Guasti L, Paul A, Laufer E \& King P 2010 Localization of Sonic hedgehog secreting and receiving cells in the developing and adult rat adrenal cortex. Molecular and Cellular Endocrinology 336 117-122. (doi:10.1016/j.mce.2010.11.010)

Karpac J, Ostwald D, Bui S, Hunnewell P, Shankar M \& Hochgeschwender U 2005 Development, maintenance, and function of the adrenal gland in early postnatal proopiomelanocortin-null mutant mice. Endocrinology 146 2555-2562. (doi:10.1210/en.2004-1290)

Karpac J, Kern A \& Hochgeschwender U 2007 Pro-opiomelanocortin peptides and the adrenal gland. Molecular and Cellular Endocrinology 265-266 29-33. (doi:10.1016/j.mce.2006.12.035)

Lefkowitz RJ, Roth J, Pricer W \& Pastan I 1970 ACTH receptors in the adrenal: specific binding of ACTH-125I and its relation to adenyl cyclase. PNAS 65 745-752. (doi:10.1073/pnas.65.3.745)

Lehoux JG, Fleury A \& Ducharme L 1998 The acute and chronic effects of adrenocorticotropin on the levels of messenger ribonucleic acid and protein of steroidogenic enzymes in rat adrenal in vivo. Endocrinology 139 3913-3922. (doi:10.1210/en.139.9.3913)

Metherell LA, Chapple JP, Cooray S, David A, Becker C, Ruschendorf F, Naville D, Begeot M, Khoo B, Nurnberg P et al. 2005 Mutations in MRAP, encoding a new interacting partner of the ACTH receptor, cause familial glucocorticoid deficiency type 2. Nature Genetics 37 166-170. (doi:10.1038/ng1501)

Mitani F, Mukai K, Miyamoto H, Suematsu M \& Ishimura Y 2003 The undifferentiated cell zone is a stem cell zone in adult rat adrenal cortex. Biochimica et Biophysica Acta 1619 317-324. (doi:10.1016/S0304-4165(02)00490-7)

Robinson PM, Comline RS, Fowden AL \& Silver M 1983 Adrenal cortex of fetal lamb: changes after hypophysectomy and effects of Synacthen on cytoarchitecture and secretory activity. Quarterly Journal of Experimental Physiology 68 15-27.

Roy S, Rached M \& Gallo-Payet N 2007 Differential regulation of the human adrenocorticotropin receptor [melanocortin-2 receptor (MC2R)] by human MC2R accessory protein isoforms alpha and beta in isogenic human embryonic kidney 293 cells. Molecular Endocrinology 21 1656-1669. (doi:10.1210/me.2007-0041)

Sebag JA \& Hinkle PM 2007 Melanocortin-2 receptor accessory protein MRAP forms antiparallel homodimers. PNAS 104 20244-20249. (doi:10.1073/pnas.0708916105)

Sebag JA \& Hinkle PM 2009 Regions of melanocortin 2 (MC2) receptor accessory protein necessary for dual topology and MC2 receptor trafficking and signaling. Journal of Biological Chemistry 284 610-618. (doi:10.1074/jbc.M804413200)

Sebag JA \& Hinkle PM 2010 Regulation of G protein-coupled receptor signaling: specific dominant-negative effects of melanocortin 2 receptor accessory protein 2. Science Signaling 3 ra28. (doi:10.1126/ scisignal.2000593)

Wright N \& Voncina D 1977 Studies on the postnatal growth of the rat adrenal cortex. Journal of Anatomy 123 147-156.

Received in final form 21 February 2011

Accepted 2 March 2011

Made available online as an Accepted Preprint 2 March 2011 